\title{
Riego con incremento de gasto en surcos cerrados de corta longitud y fuerte pendiente
}

\author{
E. Vázquez-Fernández \\ Instituto de Ingeniería, UNAM \\ Email: ernes@servidor.unam.mx
}

(recibido: abril de 2000; aceptado: julio de 2000)

\begin{abstract}
Resumen
Se presentan resultados de uniformidad de distribución teórica, los cuales se obtuvieron en surcos cerrados de corta longitud y fuerte pendiente al emplear el riego con incremento de gasto. Se hace una comparación con las calculadas mediante los riegos intermitentes y continuos. Se muestra que las uniformidades de distribución de los dos primeros métodos son muy aproximadas y superiores a las del riego continuo; sin embargo, siendo más simple y económico en su aplicación el riego con incremento de gasto, el método es factible y puede emplearse en el campo para propiciar así el ahorro de agua para el riego.
\end{abstract}

Descriptores: riego con incremento de gasto, riego continuo, riego intermitente, uniformidad de distribución.

\section{Abstract}

Results of theoretical distribution uniformity are presented which were derived from closed furrows having short length and steep gradient by employing irrigation with flow discharge increase. A comparison is made with values calculated from intermittent and continuous irrigation. It can be shown that the distribution uniformity of the two first methods is quite approximate and better than that of the continuous irrigation. However, since irrigation with discharge increase is simpler and more economic in its application, it represents a feasible solution and can be used in the field to induce savings in water for irrigation purposes.

Keywords: irrigation with discharge rate increase; continuous irrigation; intermittent irrigation; uniformity of distribution.

\section{Introducción}

El riego continuo $(\mathrm{RC})$ es el método de riego tradicional en surcos cerrados que, cuando está bien diseñado, produce eficiencias de aplicación hasta del $90 \%$ (Rendón et al.,1995). Sin embargo, cuando las pendientes son fuertes, la eficiencia se reduce al 70 u $80 \%$ (Vázquez y De la Cruz, 1998). Por otra parte, el riego intermitente (RI) presenta eficiencias más altas pero requiere de un dispositivo para crear la intermitencia del flujo; por ejemplo, el "diabeto" (Martínez y Aldama, 1998). En cambio, el riego con incremento de gasto (RIG) sólo requiere de un manejo adecuado del agua para tener eficiencias muy aproximadas a las del RI.

El RIG en surcos cerrados (Vázquez, 2000) es un método que ha mostrado en la teoría, através de simulaciones numéricas con un modelo hidrodinámico completo (Vázquez y Gracia, 1998), el incremento a la uniformidad de distribución (UD) del riego continuo.
En este trabajo, se hace una comparación de las eficiencias de aplicación o uniformidades de distribución de los tres métodos con el empleo de dicho modelo en surcos cortos de fuerte pendiente, como podrían ser los que se presentan en huertos familiares. El objetivo es demostrar que el RIG compite en el ahorro de agua con el riego intermitente, pero a un costo más bajo, ya que no es necesario emplear un dispositivo hidráulico adicional.

\section{Riego con incremento de gasto}

El procedimiento para aplicar el RIG consiste en regar una batería completa de surcos con un gasto constante hasta que termina la etapa de avance del agua (tiempo que tarda en llegar el agua desde la cabecera al extremo inferior del surco), como en el riego continuo. Después, el gasto de alimentación constante se aplica en una mitad de la batería de surcos, lo que hace que el gasto se incremente al doble hasta un tiempo de riego determinado previamente para 
DOI: http://dx.doi.org/10.22201/fi.25940732e.2000.01n4.019

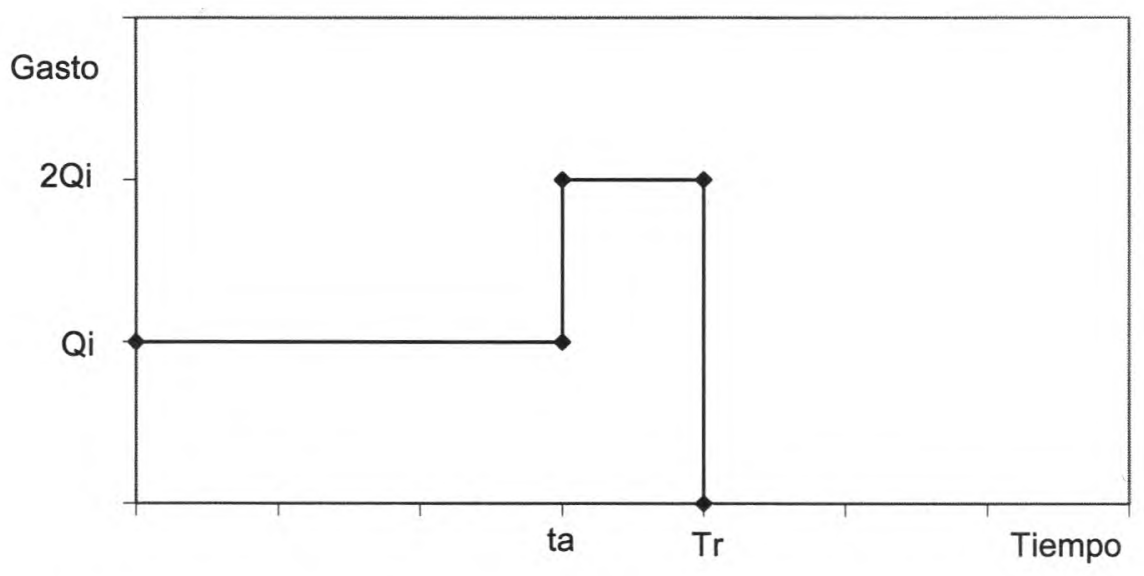

Figura 1. Hidrograma para el riego con incremento de gasto normal

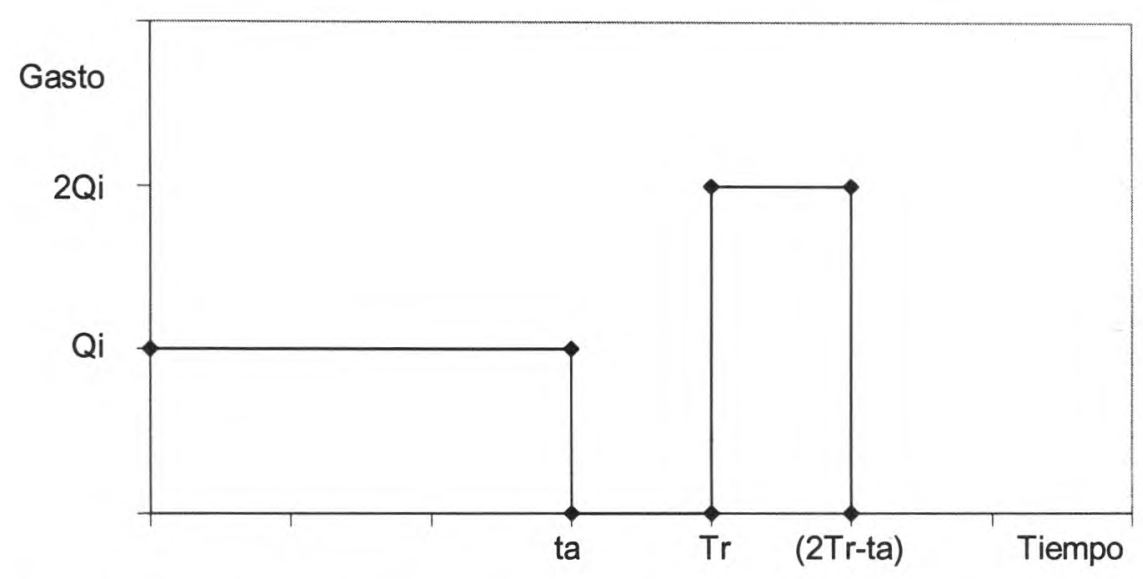

Figura 2. Hidrograma para el riego con incremento de gasto discontinuo

infiltrar la dosis de diseño, que generalmente, se presenta en torno a $2 / 3$ de la longitud del surco en las simulaciones numéricas; posteriormente, se aplica el gasto constante en la otra mitad de la parcela, dejándose de regar la primera hasta un tiempo adicional igual a la diferencia del tiempo de riego de la primera mitad menos la duración de la etapa de avance, con ello, también en la segunda mitad de surcos, el gasto se incrementa al doble. En las figuras 1 y 2 se muestran los hidrogramas de entrada en las mitades de la batería de surcos; en la figura 1, Qi es el gasto inicial, ta es la duración de la etapa de avance y $\mathrm{Tr}$ el tiempo de riego; en la figura 2, siendo Qi el mismo gasto inicial, se observa que el riego es con incremento de gasto discontinuo y que el tiempo de riego ( $2 \mathrm{Tr}$-ta) de la batería de surcos se alcañza después de la discontinuidad. El número de surcos en cada batería se determina de acuerdo con el gasto disponible en la parcela.

La relación conveniente entre el tiempo de riego $(T r)$ y la duración de la etapa de avance (ta) para producir la máxima UD es 1.33 (Vázquez, 2000). Esta relación es la mostrada en las figuras 1 y 2 . La UD se calcula como la relación $\mathrm{Zm} / \mathrm{Zp}$, donde $\mathrm{Zm}$ es la lámina mínima infiltrada, y $\mathrm{Zp}$ el promedio de ellas a lo largo del surco. Cuando se satisface la dósis o la lámina mínima requerida por la planta en surcos cerrados, la eficiencia de aplicación es idéntica a la uniformidad de distribución (Burt et al., 1997).

El riesgo de aplicar este método es que se produzcan desbordamientos en los surcos donde se aplica el RIG normal (figura 1), por lo que en las simulaciones numéricas siempre debe verificarse que el tirante máximo en la cola del surco sea menor que la profundidad del mismo.

\section{Resultados}

Para comprobar que la UD del RIG es muy aproximada a la del RI, se realizaron simulaciones numéricas en 28 surcos de forma parabólica (Vázquez, 2000) cuyos datos principales aparecen en la tabla 1. Nótese que las longitudes de los surcos son menores de $100 \mathrm{~m}$, las pendientes mayores de 


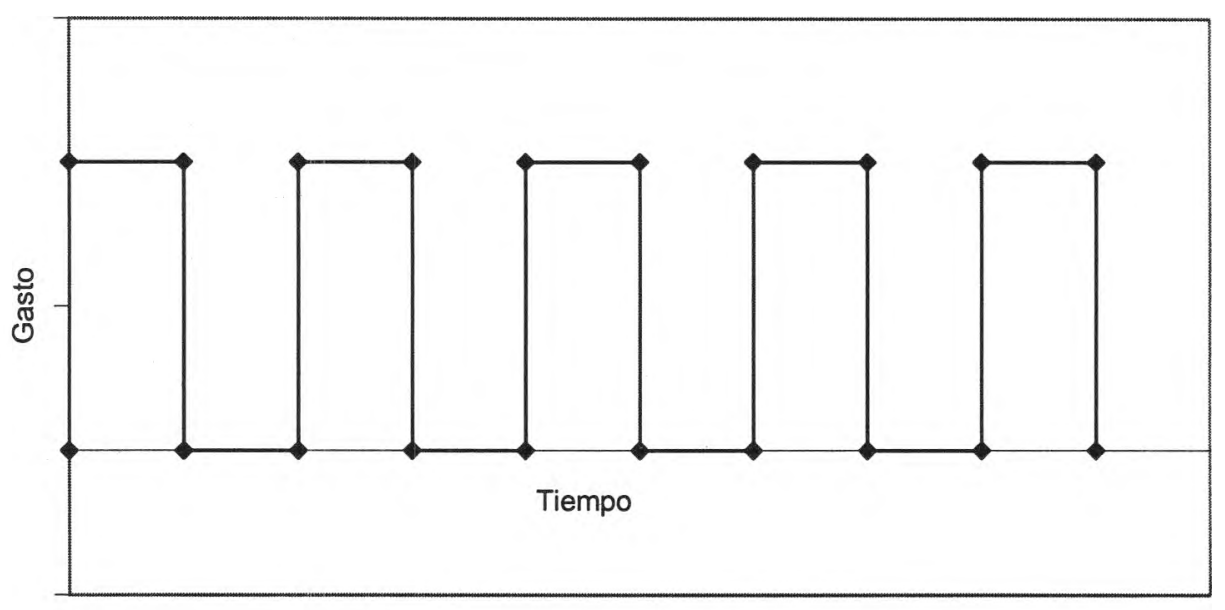

Figura 3. Hidrograma para el riego intermitente

$0.15 \%$, la gama de conductividades hidráulicas amplia las dosis mayores de $5 \mathrm{~cm}$. Cabe aclarar que para cualquier valor de la dosis, los resultados en las UD son semejantes. En las tablas 2, 3 y 4 se muestran resultados para el RC (con gasto igual al inicial del RIG), RIG y RI, respectivamente. En ninguno de los ejemplos del RIG (tabla 3 ) el tirante máximo en la cola del surco fue mayor a la profundidad del mismo.

En el RI se supone que el gasto se alterna de un surco a otro (o de una mitad a otra de la parcela) con el mismo caudal, como se indica en la figura 3, por lo que el gasto de diseño es del doble al correspondiente del RC (o inicial del RIG). En la figura 4 se concentran de manera gráfica, para una mejor visualización, los resultados de las UD de las tablas 2, 3 y 4; ahí se anota en el eje horizontal la conductividad hidráulica como parámetro más represen. tativo de los surcos. Véase que las UD del RI y el RIG son muy aproximadas. Por último, en la figura 5 se muestran las láminas infiltradas al final del proceso de riego para el surco 28 .

Tabla 1. Datos principales de los surcos

\begin{tabular}{ccccccc}
\hline $\begin{array}{c}\text { No. } \\
\text { Surco }\end{array}$ & $\begin{array}{c}\text { Pendiente } \\
\%\end{array}$ & $\begin{array}{c}\text { Longitud } \\
(\mathrm{m})\end{array}$ & $\begin{array}{c}\text { Profundidad } \\
(\mathrm{cm})\end{array}$ & $\begin{array}{c}\text { Separación } \\
(\mathrm{cm})\end{array}$ & $\begin{array}{c}\text { Conductividad } \\
\text { hidráulica }(\mathrm{cm} / \mathrm{min})\end{array}$ & $\begin{array}{c}\text { Dosis } Z \mathrm{~m} \\
(\mathrm{~cm})\end{array}$ \\
\hline 1 & 0.1500 & 90 & 30 & 73.4 & 0.013 & 6.5 \\
2 & 0.1575 & 88 & 25 & 70.4 & 0.017 & 6.1 \\
3 & 0.1631 & 86 & 24 & 92.7 & 0.019 & 5.7 \\
4 & 0.1688 & 84 & 30 & 75.0 & 0.022 & 5.9 \\
5 & 0.1744 & 82 & 30 & 73.4 & 0.024 & 5.8 \\
6 & 0.1800 & 80 & 25 & 70.4 & 0.027 & 5.5 \\
7 & 0.1856 & 78 & 23 & 89.9 & 0.030 & 5.0 \\
8 & 0.1913 & 76 & 30 & 75.0 & 0.032 & 5.2 \\
9 & 0.1962 & 74 & 23 & 89.9 & 0.035 & 5.5 \\
10 & 0.2025 & 72 & 26 & 66.4 & 0.038 & 5.7 \\
11 & 0.2081 & 70 & 30 & 75.0 & 0.040 & 6.6 \\
12 & 0.2188 & 68 & 26 & 72.2 & 0.043 & 6.0 \\
13 & 0.2194 & 66 & 23 & 89.9 & 0.045 & 5.9 \\
14 & 0.2250 & 64 & 26 & 66.4 & 0.048 & 7.4 \\
15 & 0.2306 & 62 & 30 & 75.0 & 0.051 & 5.3 \\
16 & 0.2363 & 60 & 26 & 72.2 & 0.053 & 5.1 \\
17 & 0.2419 & 58 & 23 & 89.9 & 0.056 & 5.4 \\
\hline \hline
\end{tabular}


DOI: http://dx.doi.org/10.22201/fi.25940732e.2000.01n4.019

Tabla 1. Datos principales de los surcos (continuación)

\begin{tabular}{ccccccc}
\hline $\begin{array}{c}\text { No. } \\
\text { Surco }\end{array}$ & $\begin{array}{c}\text { Pendiente } \\
(\%)\end{array}$ & $\begin{array}{c}\text { Longitud } \\
(\mathrm{m})\end{array}$ & $\begin{array}{c}\text { Profundidad } \\
(\mathrm{cm})\end{array}$ & $\begin{array}{c}\text { Separación } \\
(\mathrm{cm})\end{array}$ & $\begin{array}{c}\text { Conductividad } \\
\text { hidráulica }(\mathrm{cm} / \mathrm{min})\end{array}$ & $\begin{array}{c}\text { Dosis Zm } \\
(\mathrm{cm})\end{array}$ \\
\hline \hline 18 & 0.2475 & 56 & 28 & 69.1 & 0.059 & 5.4 \\
19 & 0.2531 & 54 & 30 & 75.0 & 0.061 & 5.6 \\
20 & 0.2588 & 52 & 26 & 72.2 & 0.064 & 7.0 \\
21 & 0.2644 & 50 & 23 & 89.9 & 0.066 & 5.5 \\
22 & 0.2700 & 48 & 25 & 64.6 & 0.069 & 5.4 \\
23 & 0.2756 & 46 & 30 & 75.0 & 0.072 & 6.1 \\
24 & 0.2813 & 44 & 25 & 70.4 & 0.074 & 6.8 \\
25 & 0.2869 & 42 & 23 & 89.9 & 0.077 & 5.2 \\
26 & 0.2925 & 40 & 26 & 66.4 & 0.080 & 5.7 \\
27 & 0.3000 & 38 & 25 & 70.4 & 0.083 & 6.6 \\
28 & 0.1800 & 95 & 23 & 65.0 & 0.040 & 15.0 \\
\hline \hline
\end{tabular}

Tabla 2. Resultados y uniformidad de distribución para el RC

\begin{tabular}{|c|c|c|c|c|c|}
\hline $\begin{array}{l}\text { No. } \\
\text { Surco }\end{array}$ & $\begin{array}{c}\text { Gasto inicial } \\
(1 / \mathrm{s})\end{array}$ & $\begin{array}{c}\text { Duración } \\
\text { etapa de avance (min) }\end{array}$ & $\begin{array}{c}\text { Tiempo de riego } \\
(\mathrm{min})\end{array}$ & $\begin{array}{l}\text { Tirante máximo } \\
(\mathrm{cm})\end{array}$ & $\begin{array}{l}\text { UD } \\
(\%)\end{array}$ \\
\hline 1 & 2.00 & 24 & 42 & 10.3 & 87 \\
\hline 2 & 1.90 & 23 & 37 & 9.3 & 88 \\
\hline 3 & 1.85 & 28 & 48 & 8.7 & 85 \\
\hline 4 & 1.80 & 24 & 41 & 8.2 & 85 \\
\hline 5 & 1.72 & 23 & 40 & 9.5 & 85 \\
\hline 6 & 1.67 & 21 & 36 & 8.9 & 87 \\
\hline 7 & 1.60 & 24 & 43 & 8.1 & 83 \\
\hline 8 & 1.57 & 22 & 38 & 7.6 & 84 \\
\hline 9 & 1.30 & 33 & 58 & 8.1 & 83 \\
\hline 10 & 1.30 & 24 & 42 & 8.7 & 84 \\
\hline 11 & 1.10 & 35 & 62 & 7.5 & 85 \\
\hline 12 & 1.00 & 34 & 58 & 8.1 & 86 \\
\hline 13 & 0.90 & 44 & 78 & 7.6 & 83 \\
\hline 14 & 0.85 & 41 & 74 & 8.8 & 85 \\
\hline 15 & 0.90 & 32 & 55 & 6.6 & 82 \\
\hline 16 & 0.85 & 30 & 52 & 7.5 & 86 \\
\hline 17 & 0.75 & 43 & 77 & 7.1 & 83 \\
\hline 18 & 0.75 & 32 & 57 & 7.8 & 85 \\
\hline 19 & 0.70 & 38 & 66 & 6.7 & 85 \\
\hline 20 & 0.60 & 49 & 86 & 7.9 & 88 \\
\hline 21 & 0.60 & 48 & 84 & 6.9 & 84 \\
\hline 22 & 0.60 & 32 & 56 & 7.3 & 85 \\
\hline 23 & 0.55 & 44 & 77 & 6.4 & 86 \\
\hline 24 & 0.50 & 48 & 82 & 7.4 & 89 \\
\hline 25 & 0.50 & 46 & 80 & 6.5 & 86 \\
\hline 26 & 0.50 & 35 & 60 & 7.2 & 87 \\
\hline 27 & 0.45 & 45 & 76 & 7.1 & 90 \\
\hline 28 & 1.60 & 63 & 112 & 10.4 & 87 \\
\hline
\end{tabular}


DOI: http://dx.doi.org/10.22201/fi.25940732e.2000.01n4.019

Tabla 3. Resultados y uniformidad de distribución para el RIG normal $(\mathrm{Tr} / \mathrm{ta}=1.33)$

\begin{tabular}{|c|c|c|c|c|c|c|c|}
\hline $\begin{array}{l}\text { No. } \\
\text { Surco }\end{array}$ & $\begin{array}{l}\text { Gasto inicial } \\
\quad(1 / s)\end{array}$ & $\begin{array}{l}\text { Duración etapa } \\
\text { de avance (min) }\end{array}$ & $\begin{array}{l}\text { Tiempo de } \\
\text { riego (min) }\end{array}$ & $\begin{array}{l}\text { Tirante máximo } \\
(\mathrm{cm})\end{array}$ & $\begin{array}{l}\text { Borde libre } \\
(\mathrm{cm})\end{array}$ & $\begin{array}{l}\text { UD } \\
\text { (\%) }\end{array}$ & $\begin{array}{l}\text { Incremento de } \\
\text { UD con respecto } \\
\text { al RC (\%) }\end{array}$ \\
\hline 1 & 2.00 & 24 & 31 & 12.5 & 17.5 & 91 & 4 \\
\hline 2 & 1.90 & 23 & 30 & 12.4 & 12.6 & 89 & 1 \\
\hline 3 & 1.85 & 28 & 37 & 11.5 & 12.5 & 88 & 3 \\
\hline 4 & 1.80 & 24 & 32 & 11.0 & 19.4 & 88 & 3 \\
\hline 5 & 1.72 & 23 & 31 & 12.3 & 17.7 & 89 & 4 \\
\hline 6 & 1.67 & 21 & 28 & 11.8 & 13.2 & 88 & 1 \\
\hline 7 & 1.60 & 24 & 33 & 10.7 & 12.3 & 86 & 3 \\
\hline 8 & 1.57 & 22 & 29 & 10.3 & 19.7 & 86 & 2 \\
\hline 9 & 1.30 & 32 & 43 & 11.1 & 11.9 & 88 & 5 \\
\hline 10 & 1.30 & 24 & 32 & 11.7 & 14.3 & 88 & 4 \\
\hline 11 & 1.10 & 35 & 47 & 11.0 & 19.0 & 89 & 4 \\
\hline 12 & 1.00 & 34 & 45 & 12.0 & 14.0 & 89 & 3 \\
\hline 13 & 0.90 & 44 & 59 & 11.2 & 11.8 & 89 & 6 \\
\hline 14 & 0.85 & 41 & 55 & 12.6 & 13.4 & 91 & 6 \\
\hline 15 & 0.90 & 32 & 43 & 10.1 & 19.9 & 88 & 6 \\
\hline 16 & 0.85 & 30 & 40 & 11.1 & 14.9 & 89 & 3 \\
\hline 17 & 0.75 & 43 & 58 & 10.8 & 12.2 & 89 & 6 \\
\hline 18 & 0.75 & 32 & 43 & 11.6 & 16.3 & 90 & 5 \\
\hline 19 & 0.70 & 38 & 50 & 10.2 & 19.8 & 89 & 4 \\
\hline 20 & 0.60 & 49 & 65 & 12.1 & 13.9 & 92 & 4 \\
\hline 21 & 0.60 & 48 & 64 & 10.7 & 12.3 & 90 & 6 \\
\hline 22 & 0.60 & 32 & 43 & 10.8 & 14.2 & 90 & 5 \\
\hline 23 & 0.55 & 44 & 58 & 10.2 & 19.8 & 90 & 4 \\
\hline 24 & 0.50 & 48 & 64 & 11.6 & 13.4 & 92 & 3 \\
\hline 25 & 0.50 & 46 & 61 & 10.2 & 12.8 & 91 & 5 \\
\hline 26 & 0.50 & 35 & 46 & 10.8 & 15.2 & 92 & 5 \\
\hline 27 & 0.45 & 45 & 60 & 11.3 & 13.7 & 92 & 2 \\
\hline 28 & 1.60 & 63 & 84 & 15.4 & 7.2 & 92 & 5 \\
\hline
\end{tabular}

Tabla 4. Resultados y uniformidad de distribución para el RI

\begin{tabular}{cccccc}
\hline $\begin{array}{c}\text { No. } \\
\text { Surco }\end{array}$ & $\begin{array}{c}\text { Gasto } \\
(1 / \mathrm{s})\end{array}$ & $\begin{array}{c}\text { Duración etapa de } \\
\text { avance }(\mathrm{min})\end{array}$ & $\begin{array}{c}\text { Tiempo de riego } \\
(\mathrm{min})\end{array}$ & $\begin{array}{c}\text { UD } \\
(\%)\end{array}$ & $\begin{array}{c}\text { Incremento de UD con } \\
\text { respecto al RIG (\%) }\end{array}$ \\
\hline 1 & 4.00 & 18 & 38 & 92 & 1 \\
2 & 3.80 & 16 & 36 & 91 & 2 \\
3 & 3.70 & 19 & 46 & 90 & 2 \\
4 & 3.60 & 17 & 38 & 91 & 3 \\
5 & 3.44 & 16 & 39 & 90 & 3 \\
6 & 3.33 & 15 & 35 & 91 & 3 \\
7 & 3.20 & 16 & 40 & 88 & 3 \\
8 & 3.14 & 15 & 34 & 89 & \\
9 & 2.60 & 20 & 54 & 39 & \\
\hline
\end{tabular}


DOI: http://dx.doi.org/10.22201/fi.25940732e.2000.01n4.019

Tabla 4. Resultados y uniformidad de distribución para el RI (continuación)

\begin{tabular}{cccccc}
\hline $\begin{array}{c}\text { No. } \\
\text { Surco }\end{array}$ & $\begin{array}{c}\text { Gasto } \\
(1 / \mathrm{s})\end{array}$ & $\begin{array}{c}\text { Duración etapa de } \\
\text { avance (min) }\end{array}$ & $\begin{array}{c}\text { Tiempo de riego } \\
(\mathrm{min})\end{array}$ & $\begin{array}{c}\text { UD } \\
(\%)\end{array}$ & $\begin{array}{c}\text { Incremento de UD con } \\
\text { respecto al RIG (\%) }\end{array}$ \\
\hline \hline 11 & 1.10 & 35 & 62 & 85 & 1 \\
12 & 2.00 & 20 & 53 & 91 & 2 \\
13 & 1.80 & 25 & 72 & 89 & 0 \\
14 & 1.70 & 26 & 68 & 91 & 0 \\
15 & 1.80 & 17 & 50 & 88 & 0 \\
16 & 1.70 & 16 & 46 & 90 & 0 \\
17 & 1.50 & 23 & 70 & 88 & 0 \\
18 & 1.50 & 18 & 52 & 89 & 0 \\
19 & 1.40 & 20 & 59 & 94 & 0 \\
20 & 1.20 & 26 & 78 & 90 & 0 \\
21 & 1.20 & 23 & 75 & 90 & 1 \\
2 & 1.20 & 17 & 50 & 91 & 2 \\
223 & 1.10 & 21 & 70 & 94 & 0 \\
425 & 1.00 & 23 & 74 & 90 & 2 \\
26 & 1.00 & 20 & 73 & 91 & 0 \\
28 & 1.00 & 18 & 56 & 94 & \\
\hline
\end{tabular}

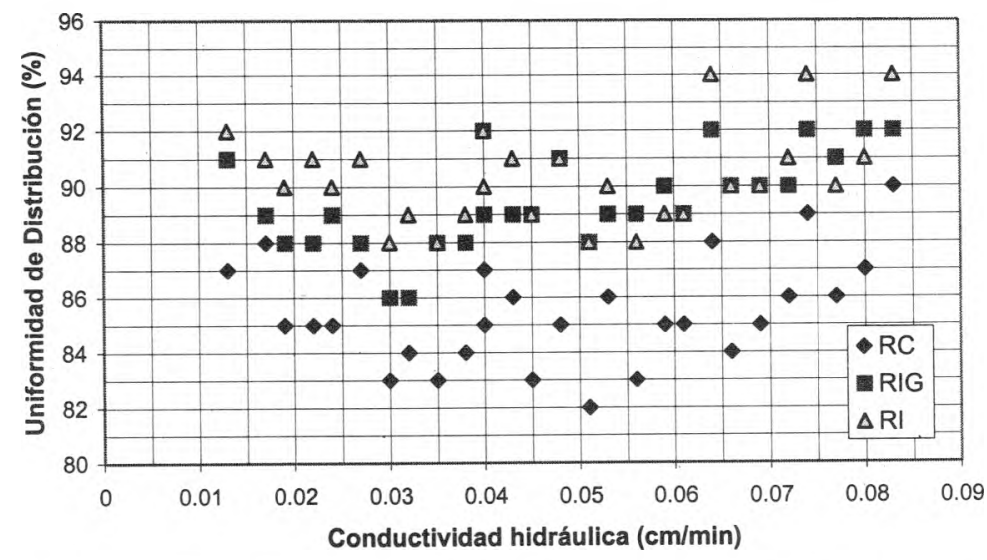

Figura 4. Uniformidades de distribución en los riegos $R C, R I$ y $R I G$

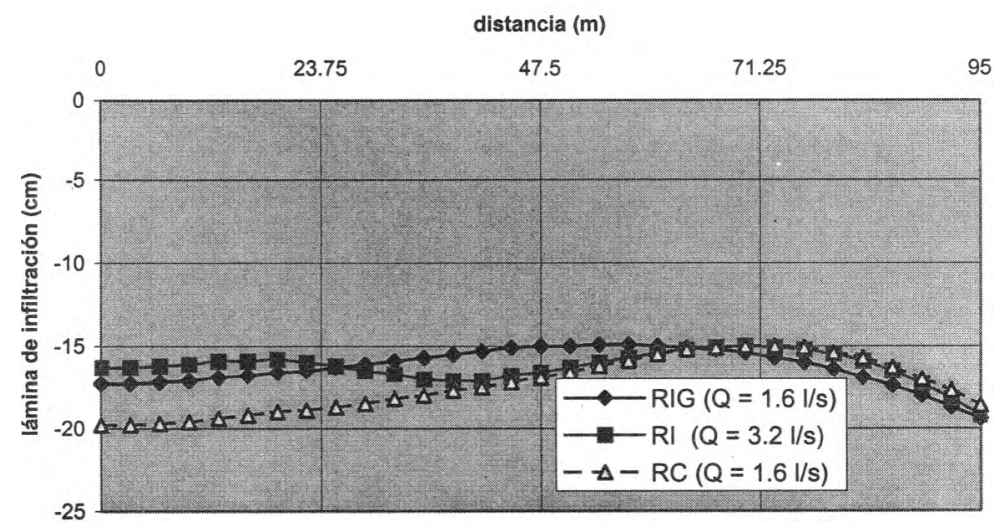

Figura 5. Láminas infiltradas en el surco No.28 


\section{Conclusiones}

Según los resultados mostrados en surcos cerrados cortos y de fuerte pendiente, las UD del RC son menores a las del RIG y RI. El segundo método supera al primero en ese indicador hasta un $6 \%$ (tabla 3 ) y el tercero supera al segundo hasta un $3 \%$ en 3 de 28 casos; y finalmente son iguales en 12 de 28. Eso permite concluir que, si el manejo del agua es adecuado, el empleo del RIG es conveniente, pues no requiere de dispositivo alguno y supera la UD del RC tanto como el RI.

\section{Referencias}

Burt C.M., Clemens A.J., Strelkoff T.S., Solomon K.H., Bliesner R.D., Hardy L.A., Howell T.A., Eisenhauer D.E. (1997). Irrigation Performance Measures: Efficiency and
Uniformity. Journal of Irrigation and Drainage Enginneering, Vol. 123, No.6, p.431.

Martínez P. y Aldama A. (1998). Fluid Device for Surge Flow Irrigation. Journal of Irrigation and Drainage Enginneering, Vol. 124, No. 5.

Rendón P.L., Fuentes R.C. y Magaña S.G. (1995). Diseño simplificado del riego por gravedad. Memorias del VI Congreso Nacional de Irrigación. Chapingo, México.

Vázquez E. (2000). Diseño del riego con incremento de gasto en surcos cerrados. Ingeniería del agua, Universidad Politécnica de Valencia, España (en prensa).

Vázquez E. y Gracia J. (1998). Calibración de parámetros de infiltración y rugosidad con un modelo numérico para riego con surcos cerrados. Ingeniería del agua, Vol. 5, No. 3. Universidad Politécnica de Valencia, España. Vázquez E. y De la Cruz R. (1998). Criterio de diseño para el riego por gravedad con surcos cerrados. Ingeniería. Investigación y Tecnología, Vol.I, No.1, UNAM, p.38. 